\title{
Extended Scaling for Ferromagnets
}

\author{
I. A. Campbell, ${ }^{1}$ K. Hukushima, ${ }^{2}$ and H. Takayama ${ }^{3}$ \\ ${ }^{1}$ Laboratoire des Colloïdes, Verres et Nanomatériaux, \\ Université Montpellier II, 34095 Montpellier, France \\ 2 Department of Basic Science, University of Tokyo, Tokyo, 153-8902, Japan \\ ${ }^{3}$ Institute for Solid State Physics, University of Tokyo, \\ Kashiwa-no-ha 5-1-5, Kashiwa, 27r-8581, Japan
}

(Dated: April 3, 2018)

\begin{abstract}
A simple systematic rule, inspired by high-temperature series expansion (HTSE) results, is proposed for optimizing the expression for thermodynamic observables of ferromagnets exhibiting critical behavior at $T_{\mathrm{c}}$. This "extended scaling" scheme leads to a protocol for the choice of scaling variables, $\tau=\left(T-T_{\mathrm{c}}\right) / T$ or $\left(T^{2}-T_{\mathrm{c}}^{2}\right) / T^{2}$ depending on the observable instead of $\left(T-T_{\mathrm{c}}\right) / T_{\mathrm{c}}$, and more importantly to temperature dependent non-critical prefactors for each observable. The rule corresponds to scaling of the leading of the reduced susceptibility above $T_{\mathrm{c}}$ as $\chi_{\mathrm{c}}^{*}(T) \sim \tau^{-\gamma}$ in agreement with standard practice with scaling variable $\tau$, and for the leading term of the second-moment correlation length as $\xi_{\mathrm{c}}^{*}(T) \sim T^{-1 / 2} \tau^{-\nu}$. For the specific heat in bipartite lattices the rule gives $C_{\mathrm{c}}^{*}(T) \sim T^{-2}\left[\left(T^{2}-T_{\mathrm{c}}^{2}\right) / T^{2}\right]^{-\alpha}$. The latter two expressions are not standard. The scheme can allow for confluent and non-critical correction terms. A stringent test of the extended scaling is made through analyses of high precision numerical and HTSE data, or real data, on the three-dimensional canonical Ising, XY, and Heisenberg ferromagnets. For the susceptibility $\chi(T)$ and the correlation length $\xi(T)$ of the three ferromagnets, their optimized expression, which consists of the leading term (respectively $\chi_{\mathrm{c}}^{*}(T)$ and $\xi_{\mathrm{c}}^{*}(T)$ ) and a quite limited number of confluent and non-critical correction terms, represents real data to surprisingly good approximations over the entire temperature range from $T_{\mathrm{c}}$ to infinity. The temperature dependent prefactors introduced are of crucial importance not only in fixing the optimized expression at relatively high temperatures but also in determining appropriately the small amplitude correction terms. For the specific heat of the Ising ferromagnet, $C_{\mathrm{c}}^{*}(T)$ combined with two non-critical correction terms which are calculated with no free parameters once the correlation length critical parameters are known, reproduces real data nicely also over the whole temperature range.
\end{abstract}

PACS numbers: 75.50.Lk, 75.40.Mg, 05.50.+q

\section{INTRODUCTION}

At a continuous transition, the expression $F_{c}^{*}(T)$ for the leading critical behavior of a thermodynamic observable $F(T)$ has the well known form

$$
F_{\mathrm{c}}^{*}(T) \sim\left(T-T_{\mathrm{c}}\right)^{-\rho_{F}},
$$

where $T_{\mathrm{c}}$ and $\rho_{F}$ are the transition temperature and the critical exponent respectively. For the concrete analysis of numerical data, a normalization factor with noncritical behavior at $T_{\mathrm{c}}$ must be introduced. The simplest and most traditional convention, which will be referred to below as $T$ scaling, is to normalize each $F_{\mathrm{c}}^{*}(T)$ by a temperature independent constant. For obvious reasons this constant is chosen to be $T_{\mathrm{c}}^{\rho_{F}}$ for each observable; one then writes the normalized leading term as the familiar text-book expression:

$$
F_{\mathrm{c}}^{*}(T)=\mathcal{C}_{F}\left[\left(T-T_{\mathrm{c}}\right) / T_{\mathrm{c}}\right]^{-\rho_{F}}=\mathcal{C}_{F} t^{-\rho_{F}},
$$

where $t=\left(T-T_{\mathrm{c}}\right) / T_{\mathrm{c}}$ and $\mathcal{C}_{F}$ is the critical amplitude (see [1] for a detailed review). An alternative and a priori equally valid choice is to write

$$
\begin{aligned}
F_{\mathrm{c}}^{*}(\beta) & =\mathcal{C}_{F}\left[\left(\beta_{c}-\beta\right) / \beta_{c}\right]^{-\rho_{F}}=\mathcal{C}_{F}\left[\left(T-T_{\mathrm{c}}\right) / T\right]^{-\rho_{F}} \\
& =\mathcal{C}_{F}\left[1-\frac{\beta}{\beta_{\mathrm{c}}}\right]^{-\rho_{F}}=\mathcal{C}_{F} \tau^{-\rho_{F}},
\end{aligned}
$$

where $\beta$ is the inverse temperature $1 / T$ and $\tau=1-\beta / \beta_{\mathrm{c}}$. Note that the temperature dependence of the normalization is now different for each observable. This " $\beta$ scaling" form has become the standard normalization for theoretical work on the critical properties of ferromagnets and analogous systems, see for instance $\frac{1,2,3}{2}$, although more complex normalizations have been used in special cases. At higher order, confluent and analytic correction terms (such as temperature independent constants) are introduced. Thus including the confluent correction terms, the critical behavior, $F_{\mathrm{c}}(\beta)$, is written in terms of the $\beta$ scaling as

$$
\begin{aligned}
F_{\mathrm{c}}(\beta) & =F_{c}^{*}(\beta)\left(1+a_{F} \tau^{\theta}+\cdots\right) \\
& =\mathcal{C}_{F} \tau^{-\rho_{F}}\left(1+a_{F} \tau^{\theta}+\cdots\right),
\end{aligned}
$$

where $\theta=\nu \omega$ with $\omega$ being the [universal] confluent correction exponent, and $a_{F}$ is the confluent correction amplitude. In the $T$ scaling form, $\tau$ in the above equation is replaced by $t$. This critical scaling form is firmly established by field theory in the limit of temperatures very close to $T_{C} \underline{\underline{4}}$. Ratios of the $a_{F}$ for different observables are universal $\stackrel{5}{\underline{5}}$. The exponent $\theta$ is common in both scaling forms so long as $\theta<1$. However, no general argument seems to have been given which would show that either the $T$ or the $\beta$ scaling is optimal for all (or any) observables when a much wider temperature range 
is considered. Recently we have proposed an extended scaling scheme for normalizing observables such that the leading critical expressions remain good approximations right up to the trivial fixed point at infinite temperature ${ }^{6}$. Our extended scaling scheme is based on a consideration of high-temperature series expansions (HTSE), and so is naturally formulated in terms of the $\beta$ scaling. The most important ingredient of the scheme is the introduction of non-critical prefactors $\beta^{\phi_{F}}$ in the normalizations, where each exponent $\phi_{F}$ is uniquely chosen such that the normalized $F_{c}^{*}(\beta)$ tends to the correct asymptotic form in the limit $T \rightarrow \infty$.

In the present work our aim is to further develop our extended scaling scheme to include explicitly the confluent and analytical correction terms. We then validate our scheme by analyzing data for three canonical ferromagnets: the $S=1 / 2$ Ising, XY and Heisenberg models on simple cubic lattices in dimension three. These models have been intensively studied over many years and their main critical parameters: $T_{\mathrm{c}}$, the critical exponents $\rho_{F}, \theta$, and certain critical amplitudes are known to high precision. Careful accounts of studies using different complementary approaches are given for instance in Refs. [1,3,7, 8]. Accurate simulation and HTSE results have been published in the form of tabulated data. The present analyses show that the appropriately normalized leading terms are good approximations over the entire temperature range, with small but identifiable corrections due to confluent and non-critical terms. We obtain estimates of non-universal critical parameters like critical amplitudes $\mathcal{C}_{F}$ and confluent correction amplitudes $a_{F}$ from the high precision numerical data. Our extended scaling analyses are in each case entirely consistent with field theoretical and HTSE estimates of the critical parameters.

An important result of the present analysis is to demonstrate that the prefactors $\beta^{\phi_{F}}$ which have been introduced play a crucial role in extracting accurate values of the critical exponents from simulation data even in a temperature range close to $T_{\mathrm{c}}$, such as $\tau \lesssim 0.01$. In the standard scalings without the prefactors the estimates of the leading critical term and of the confluent term from analyses of numerical data turn out to be modified to order $\sim \tau$ (note $t=\tau /(1-\tau)$ ).

The same approach based on the HTSE should be directly applicable to a wide class of systems having the same intrinsic HTSE structure as the simple ferromagnets. Extensions to more complex systems such as spin glasses are in principle straightforward ${ }^{6}$.

The paper is organized as follows. In Sec. II we explain our extended scaling scheme for various thermodynamic observables, and discuss confluent corrections to scaling terms in our scheme. In Sec. III we give methods of analysis for numerical data using our extended scaling scheme. We show how they work in practice for Ising, XY and Heisenberg ferromagnets in Sec. [V] V] and VI] respectively. In Sec. VII we make concluding remarks and discuss related problems.

\section{EXTENDED SCALING SCHEME}

\section{A. Optimized expression for observables $F(\beta)$}

Let us suppose HTSE of an observable $F(\beta)$ is given by

$$
F(\beta)=a_{F, 0} \beta^{\phi_{F}}\left(1+a_{F, 1} \beta+a_{F, 2} \beta^{2}+\cdots\right) .
$$

The most important ingredient of our extended scaling scheme is then to write $F_{\mathrm{c}}(\beta)$ as

$$
F_{\mathrm{c}}(\beta)=R_{F}^{\mathrm{c}}(\tau) a_{F, 0} \beta^{\phi_{F}} \tau^{-\rho_{F}},
$$

where

$$
R_{F}^{\mathrm{c}}(\tau)=\mathcal{R}_{F}^{*}\left(1+a_{F} \tau^{\theta}+\cdots\right),
$$

with $\mathcal{R}_{F}^{*}=\mathcal{C}_{F} /\left(a_{F, 0} \beta_{\mathrm{C}}^{\phi_{F}}\right)$. In particular, the leading contribution without the confluent correction is represented as

$$
F_{\mathrm{c}}^{*}(\beta)=\mathcal{R}_{F}^{*} a_{F, 0} \beta^{\phi_{F}} \tau^{-\rho_{F}} .
$$

The idea here is to let $F_{\mathrm{c}}(\beta)$ not only represent the correct power-law divergence $\tau^{-\rho_{F}}$ with the critical amplitude $\mathcal{C}_{F}$ (and with certain confluent correction terms) at temperatures close to $T_{\mathrm{c}}$ but also have an asymptotic form consistent with the HTSE in the high temperature limit. The observable $F(\beta)$ is then approximated as

$$
F(\beta) \simeq F^{\mathrm{opt}}(\beta)=F_{\mathrm{c}}(\beta)+b_{F, 0} \beta^{\phi_{F}}\left(1+b_{F, 1} \beta+\cdots\right) .
$$

Here the second term represents the analytic (noncritical) correction term in the present scheme. Its coefficients $b_{F, 0}$ and $b_{F, i}$ are determined in such a way that Eq. (9), combined with Eqs. (6) and (7), coincides with Eq. (5) termwise as a function of $\beta$; for example,

$$
b_{F, 0}=a_{F, 0}-\beta_{\mathrm{c}}^{-\phi_{F}} \mathcal{C}_{F}\left(1+a_{F}+\cdots\right),
$$

and a similar expression for $b_{F, 1}$. The above set of equations with the minimum number of the confluent and analytic correction terms is an optimized expression we propose for the function $F(\beta)$ which is analytic in the range $0 \leq \beta<\beta_{\mathrm{c}}$ and is singular at $\beta=\beta_{\mathrm{c}}$. An important quantity for analyzing our extended scaling scheme is $R_{F}(\tau)$ defined by

$$
R_{F}(\tau)=\frac{F(\beta)}{a_{F, 0} \beta^{\phi F} \tau^{-\rho_{F}}} .
$$

It is the ratio of the measured values of observable $F(T)$ to its leading critical term including the $\beta^{\phi_{F}}$ prefactor but without the critical amplitude $\mathcal{C}_{F}$. Explicitly, in the vicinity of $T_{\mathrm{c}}$ where $F(\beta) \simeq F_{\mathrm{c}}(\beta)$, it behaves as

$$
R_{F}(\tau) \simeq R_{F}^{\mathrm{c}}(\tau) \simeq \mathcal{R}_{F}^{*}\left(1+a_{f} \tau^{\theta}+\cdots\right) .
$$

The plot $R_{F}(\tau)$ versus $\tau^{\theta}$ near $\tau=0$ thus becomes a straight line with intercept $\mathcal{R}_{F}^{*}$ and slope $\mathcal{R}_{F}^{*} a_{F}$, where the values of $T_{\mathrm{c}}, \rho_{F}$ and $\theta$ are assumed to be known ( $\phi_{F}$ and $a_{F, 0}$ are given by HTSE analysis). In the limit $\beta \rightarrow 0$, on the other hand, it becomes $R_{F}(\tau)=1+\left(a_{F, 1}-\right.$ $\left.\rho_{F} / \beta_{\mathrm{c}}\right) \beta+\cdots$. Between these limits the form of $R_{F}(\tau)$ will depend on the entire collection of unspecified higher order corrections to scaling. 


\section{B. Susceptibility}

The "true" susceptibility, naturally defined through the magnetization response to an infinitesimal applied field, is given by the fluctuation-dissipation theorem as

$$
\chi_{\mathrm{t}}(\beta)=\beta \frac{1}{N} \sum_{i j}\left\langle S_{i} S_{j}\right\rangle
$$

The reduced susceptibility $\chi_{\mathrm{red}}=\chi_{\mathrm{t}} / \beta$ is (confusingly) almost always referred to in the literature as "the susceptibility". For consistency we will follow this convention and write the reduced susceptibility as $\chi$, but we will refer systematically in the text to "reduced susceptibility".

The HTSE for the reduced susceptibility $\chi(\beta)$ in $S=$ $1 / 2$ ferromagnets is of the form with $\phi_{\chi}=0$ and $a_{\chi, 0}=1$, or with abbreviation of $a_{\chi, i}=a_{i}$,

$$
\chi(\beta)=1+a_{1} \beta+a_{2} \beta^{2}+a_{3} \beta^{3}+\cdots .
$$

Then the leading divergent expression, Eq. (8), is written as

$$
\chi_{\mathrm{c}}^{*}(\beta)=\mathcal{R}_{\chi}^{*} \tau^{-\gamma},
$$

with $\mathcal{R}_{\chi}^{*}=\mathcal{C}_{\chi}$. The ratio $R_{\chi}(\tau)$ of Eq. (11) is reduced to

$$
R_{\chi}(\tau)=\chi(\beta) / \tau^{-\gamma}\left(=\chi_{\mathrm{c}}(\beta)\right)
$$

where $\chi_{\mathrm{c}}(\beta)$ is Eq. (6) for $\chi(T)$. Note that $R_{\chi}(0)=$ $\mathcal{R}_{\chi}^{*}$ at $T_{\mathrm{c}}, R_{\chi}(\tau)=\mathcal{R}_{\chi}^{*}\left(1+a_{\chi} \tau^{\theta}+\cdots\right)$ near $T_{c}$, and $R_{\chi}(\beta)=1+\left(a_{1}-\gamma / \beta_{\mathrm{c}}\right) \beta+\cdots$ near infinite temperature. If $R_{\chi}(\tau)$ remains close to 1 over the whole temperature range (which is the case for the systems we consider as we will see below), the leading critical contribution without the correction terms, $\chi_{\mathrm{c}}^{*}(\beta)=\mathcal{R}_{\chi}^{*} \tau^{-\gamma}$, is a good approximation for the reduced susceptibility, $\chi(\beta)$. Furthermore, the small difference $\chi(\beta)-\chi_{\mathrm{c}}^{*}(\beta)$ of the Ising and XY ferromagnets in the whole temperature range $0 \leq \beta<\beta_{\mathrm{c}}$ turns out to be reproduced surprisingly well by our optimized expression, $\chi^{\text {opt }}(\beta)$ of Eq. (9), only with one confluent and two non-critical correction terms.

\section{Correlation length}

There are different alternative definitions for the correlation length, but any correlation length diverges at criticality as $\xi(T) \sim\left(T-T_{\mathrm{c}}\right)^{-\nu}$. The second moment correlation length $\xi_{\mathrm{sm}}$ is defined through the second moment

$$
\mu_{2}(\beta)=\sum_{r} r^{2}\left\langle S_{0} S_{r}\right\rangle=2 d \chi(\beta) \xi_{\mathrm{sm}}(\beta)^{2},
$$

with $d$ the space dimensionality ${ }^{3}$. From now on we will refer to $\xi_{\mathrm{sm}}(\beta)$ simply as $\xi(\beta)$. The HTSE results show that for $N$-vector $S=1 / 2$ spins, the series for $\mu_{2}(\beta)$ is of the form $B_{1} \beta+B_{2} \beta^{2}+B_{3} \beta^{3}+\cdots$ and is well behaved with
$B_{1}=z / N$, where $z$ is the number of nearest neighbors. This yields $\phi_{\xi}=1 / 2$ and $a_{\xi, 0}=(z / 2 d N)^{1 / 2}$. We then reduce Eq. (8) to

$$
\xi_{c}^{*}(\beta)=\mathcal{R}_{\xi}^{*}\left(\frac{z \beta}{2 d N}\right)^{1 / 2} \tau^{-\nu},
$$

where $\mathcal{R}_{\xi}^{*}=\mathcal{C}_{\xi} /\left(z \beta_{\mathrm{c}} / 2 d N\right)^{1 / 2}$ with $\mathcal{C}_{\xi}$ being the standard critical amplitude in Eq. (3) for $\xi$. The nonstandard normalization prefactor $\beta^{1 / 2}$ for $\xi_{c}^{*}(\beta)$ is our main result. The mean-field calculation 9 of the correlation length through the fluctuation-dissipation theorem provides an example confirming the extended scaling form of Eq. (18). See also the analysis of Fisher and Burford ${ }^{10}$, particularly their temperature dependent "effective interaction range" parameter $r_{1}(T)$.

The critically divergent part of $\xi(\beta)$ with the confluent correction terms is represented by $\xi_{\mathrm{c}}(\beta)$ and is written as

$$
\xi_{\mathrm{c}}(\beta)=\mathcal{R}_{\xi}^{*}\left(1+a_{\xi} \tau^{\theta}+\cdots\right)\left(\frac{z \beta}{2 d N}\right)^{1 / 2} \tau^{-\nu}
$$

The ratio $R_{\xi}(\tau)$ becomes

$$
R_{\xi}(\tau)=\left(\xi(\beta) /(z \beta / 2 d N)^{1 / 2}\right) / \tau^{-\nu}
$$

Again, because of the confluent correction, it becomes $R_{\xi}(\tau) \simeq \beta_{\mathrm{c}}^{1 / 2} \mathcal{R}_{\xi}^{*}\left(1+a_{\xi} \tau^{\theta}+\cdots\right)$ near $T_{c}$ and $R_{\xi}(\beta)=$ $1+\left[\left(B_{2} / 2 B_{1}\right)-\left(a_{1} / 2\right)-\nu / \beta_{\mathrm{c}}\right] \beta+\cdots$ with $a_{1}$ being the coefficient in Eq. (14) near infinite temperature.

\section{Specific heat}

The usual analysis of the specific heat (defined as the derivative of the internal energy at fixed volume $C(T)=$ $\left.d U(T) /\left.d T\right|_{V}\right)$ near criticality assumes the form

$$
C_{c}(T)=\mathcal{C}_{C}\left[\left(T-T_{c}\right) / T_{c}\right]^{-\alpha}+K
$$

where $\alpha=2-\nu d$ and $\mathcal{C}_{C}$ is the critical amplitude of $C(T)$, and it is standard practice to introduce a large non-critical (in fact temperature independent) contribution $K$ (see e.g. Ref [11]).

While the series for the reduced susceptibility and the second moment $\mu_{2}$ are polynomial functions of $\beta$ with both odd and even terms, for bipartite (such as bcc and simple cubic) lattices the HTSE expression for $C(\beta)$ consists of even powers of $\beta$ only ${ }^{12,13}$, and can be written as

$$
C(\beta)=c_{2} \beta^{2}+c_{4} \beta^{4}+c_{6} \beta^{6}+\cdots
$$

One can carry through the same type of argument ${ }^{6}$ as in the case of $\mu_{2}(T)$, except that as all the terms in the series are even in $\left(\beta / \beta_{\mathrm{c}}\right)$, the critical behavior must be re-written in terms of the scaling variable $\left[1-\left(\beta / \beta_{\mathrm{c}}\right)^{2}\right]$ replacing $\left[1-\left(\beta / \beta_{\mathrm{c}}\right)\right]$ in the equivalent expressions for the correlation length. Thus, with $\phi_{C}=2$, one can write 
the leading critical term, which corresponds to Eq. (8), as

$$
C_{\mathrm{c}}^{*}(\beta)=\beta^{2} \mathcal{R}_{C}^{*}\left(1-\left(\frac{\beta}{\beta_{\mathrm{c}}}\right)^{2}\right)^{-\alpha} \sim \frac{1}{T^{2}}\left(\frac{T-T_{\mathrm{c}}}{T}\right)^{-\alpha},
$$

where $\mathcal{R}_{C}^{*}=\mathcal{C}_{C} 2^{\alpha} / \beta_{\mathrm{c}}^{2}$. If the confluent correction terms are included, we obtain the expression corresponding to Eq. (6) as

$$
C_{\mathrm{c}}(\beta)=C_{\mathrm{c}}^{*}(\beta)\left[1+\frac{a_{C}}{2^{\theta}}\left(1-\frac{\beta^{2}}{\beta_{\mathrm{c}}^{2}}\right)^{\theta}+\cdots\right] .
$$

where $a_{C}$ is the confluent correction amplitude. It is noted that, since the two critical amplitudes $\mathcal{C}_{C}$ and $a_{C}$ are introduced in the standard way (as represented by Eq. (21) for $\mathcal{C}_{C}$ ), the factors $2^{\alpha}$ and $1 / 2^{\theta}$ appear in the definition of $\mathcal{R}_{C}^{*}$ and in Eq. (24), respectively. In fact there is a hyper-universal relationship linking this $\mathcal{C}_{C}$ to $\mathcal{C}_{\xi}^{14}$

$\mathcal{C}_{\text {hyper }}=\left(\alpha \mathcal{C}_{C}\right)^{1 / d} \mathcal{C}_{\xi}=\left(\frac{\alpha \mathcal{R}_{C}^{*}}{2^{\alpha}}\right)^{1 / d} \mathcal{R}_{\xi}^{*} \beta_{\mathrm{c}}^{1 / 2+2 / d}\left(\frac{z}{2 d}\right)^{1 / 2}$,

where $\mathcal{C}_{\text {hyper }}$ is a constant whose value is known rather accurately ${ }^{3}$. Equation (23) is not standard, but it can be seen to tend to the appropriate limit, $C_{\mathrm{c}}^{*}(T) \sim(T-$ $\left.T_{\mathrm{c}}\right)^{-\alpha}$, as $T$ approaches $T_{\mathrm{c}}$.

In practice $\mathcal{R}_{C}^{*}$ is much larger than unity (as will be seen later in the case of the $3 d$ Ising model) which is the reason for the large non-critical contribution to $C(\beta)$. The non-critical contribution is in fact not a parameter to be adjusted freely, but it has to be determined through the high temperature limit of an equation which corresponds to Eq. (9). Ignoring the confluent correction so as to clarify the discussion, we know the exact high temperature limits for $C_{\mathrm{c}}(\beta)\left(\simeq C_{\mathrm{c}}^{*}(\beta)\right)$ from Eq. (23) and for $C(\beta)$ by Eq. (22). Then $C(\beta)$ truncated to two leading non-critical correction terms is explicitly written as

$$
C(\beta)=C_{\mathrm{c}}(\beta)+\mathcal{K}_{2} \beta^{2}+\mathcal{K}_{4} \beta^{4},
$$

where the non-critical parameters $\mathcal{K}_{i}$ are given by $\mathcal{K}_{2}=$ $c_{2}-\mathcal{R}_{C}^{*}$ and $\mathcal{K}_{4}=c_{4}-\alpha \mathcal{R}_{C}^{*} / \beta_{\mathrm{c}}^{2}$. The coefficients $c_{2}$ and $c_{4}$ are known from HTSE. So if $\nu, \beta_{\mathrm{c}}$ and $R_{\xi}^{c}$ have been measured independently, we can evaluate all the parameters which one needs to fix the functional form of $C(\beta)$, such as $\alpha=2-\nu d$ and $\mathcal{R}_{C}^{*}$ determined through Eq. (25). This we assume to be $C^{\text {opt }}(\beta)$, an optimized expression for $C(\beta)$, in the whole $\beta$ range $0 \leq \beta \leq \beta_{\mathrm{c}}$. The thus calculated curve $C^{\text {opt }}(\beta)$ can be tested by comparing with simulation and HTSE data.

\section{E. Finite size scaling}

Though we will discuss thermodynamic limit behavior only and will not analyze finite-size-scaling (FSS) data explicitly in the present paper, we note for reference that the extended scaling normalization modifies the FSS expressions. The canonical FSS ansatz ${ }^{15}$ is

$$
F(T, L) \sim L^{\rho_{F} / \nu} \tilde{F}[L / \xi(T)]
$$

where $\tilde{F}(x)$ is a universal scaling function. The frequently used FSS expression derived from Eq. (27),

$$
F(T, L) \sim L^{\rho_{F} / \nu} \tilde{F}\left[L^{1 / \nu}(T-T c)\right]
$$

contains the implicit assumption of $T$ scaling for the correlation length. It is thus only appropriate if restricted to a very narrow range of temperature around $T_{c}$. With the extended scaling and the finite size correlation length $\xi(L, \beta)$, the FSS ansatz can be rewritten $\underline{6}$

$$
F(L, \beta) \sim \beta^{\phi_{F}}\left(\frac{L}{\beta^{1 / 2}}\right)^{\rho_{F} / \nu} \mathcal{F}\left[\left(\frac{L}{\beta^{1 / 2}}\right)^{1 / \nu}\left(1-\frac{\beta}{\beta_{\mathrm{c}}}\right)\right],
$$

or

$$
F(L, \beta) \sim \beta^{\phi_{F}}\left(\frac{L}{\beta^{1 / 2}}\right)^{\rho_{F} / \nu} \hat{\mathcal{F}}\left[\frac{L}{\xi(L, \beta)}\right]
$$

where the scaling functions behave as $\mathcal{F}(x) \sim x^{-\rho_{F}}$ and $\hat{\mathcal{F}}(x) \sim x^{-\rho_{F} / \nu}$ at $x \gg 1$. For the reduced susceptibility with $\phi_{\chi}=0$, the FSS form is written as

$$
\begin{aligned}
\chi(L, \beta) & \sim\left(\frac{L}{\beta^{1 / 2}}\right)^{\gamma / \nu} \mathcal{F}_{\chi}\left[\left(\frac{L}{\beta^{1 / 2}}\right)^{1 / \nu}\left(1-\frac{\beta}{\beta_{\mathrm{c}}}\right)\right] \\
& =\tau^{-\gamma} \tilde{\mathcal{F}}_{\chi}\left[\left(\frac{L}{\beta^{1 / 2}}\right)^{1 / \nu} \tau\right]
\end{aligned}
$$

where $\mathcal{F}_{\chi}(x) \sim x^{-\gamma}$ and $\tilde{\mathcal{F}}_{\chi}(x) \sim$ constant. at $x \gg 1$. In a similar manner, the FSS form for the correlation length $\xi(L, \beta)$, for which $\rho_{\xi}=\nu$ and $\phi_{\xi}=1 / 2$, is written as

$$
\xi(L, \beta) \sim L \mathcal{F}_{\xi}\left[\left(\frac{L}{\beta^{1 / 2}}\right)^{1 / \nu}\left(1-\frac{\beta}{\beta_{\mathrm{c}}}\right)\right],
$$

where $\mathcal{F}_{\xi}(x) \sim x^{-\nu}$ at $x \gg 1$. While the extended FSS scheme for the susceptibility is modified from the standard one only by the $\beta$-prefactor in the argument of $\tilde{F}_{\chi}(x)$, the scaling plot is significantly improved for $2 d$ Ising ferromagnetic and $3 d$ Ising spin glass models ${ }^{6}$.

\section{ANALYSES USING EXTENDED SCALING}

In order to make a stringent test of the extended scaling scheme, we study the three canonical ferromagnets: Ising, XY and Heisenberg, on three dimensional simple cubic lattices. High precision numerical data have been obtained for each of these systems for the temperature domain ranging from close to $T_{\mathrm{c}}$ to about $1.1 T_{\mathrm{c}}$ and the 
authors have generously published their data in tabulated form $16,17,18$. The data have been taken on systems large enough for the data points to be representative of the thermodynamic limit. Long HTSEs have also been published for $\chi$ and $\mu_{2}$ and for $C$ for all three systems ${ }^{12,19}$, and relatively longer series for the free-energy and the specific heat have been calculated for the Ising model ${ }^{13}$; these series can be used to calculate $\chi(T), \xi(T)$ and $C(T)$ explicitly for the region $T$ well above $T_{\mathrm{c}}$. Below we call these HTSE and MC data as the real data. Thanks to a combination of results from field theory and HTSE the values of the critical temperatures, the critical exponents and the critical amplitudes are known to a high degree of accuracy, and the confluent correction exponents are also well known. The [non-universal] confluent correction amplitudes are small for these three systems and the estimates are much less accurate (see Butera and Comi ${ }^{19}$ for a detailed account).

In each case we will plot the ratios $R_{\chi}(\tau)$ and $R_{\xi}(\tau)$ respectively defined by Eqs. (16) and (20) with respect to $\tau^{\theta}$, where we have used $z=2 d$ for simple cubic lattices. The plots near $\tau=0$ give us the critical and the confluent amplitudes as explained at the end of Sec. II A The ratios are defined in a way that they approach unity at infinite temperature. We will see that the two ratios defined in the extended scaling scheme are in fact close to unity (within several hundredth deviation from unity at most) in the whole range of $\tau$.

In addition, a simple scaling relation links the observables $\chi(\beta)$ and $\xi(\beta)$ through $\chi(\beta) \sim \xi^{2-\eta}(\beta)$ to leading order. This equation has the advantage that it can in principle be used to determine the exponent $\eta$ directly from a log-log plot of $\chi(\beta)$ against $\xi(\beta)$ near $\beta_{\mathrm{c}}$ without any explicit knowledge of $\beta_{\mathrm{c}}$. For the extended scaling scheme ( $\beta$ scaling with the $\beta^{\phi_{F}}$ factors), the relation can be rewritten to leading order

$$
\chi(\beta)=\frac{\chi_{\mathrm{t}}(\beta)}{\beta} \sim\left(\frac{\xi(\beta)}{\sqrt{\beta / N}}\right)^{2-\eta} .
$$

We will analyze the ratio defined by

$$
R(\tau)=\frac{\chi(\beta)}{\left(\xi(\beta) /(\beta / N)^{1 / 2}\right)^{2-\beta}} .
$$

Including the leading confluent correction factors, it behaves near $T_{c}$ as

$$
R(\tau) \simeq \mathcal{R}^{*}\left(1+\mathcal{B} \tau^{\theta}+\cdots\right)
$$

where $\mathcal{R}^{*}=\mathcal{R}_{\chi}^{*} /\left(\mathcal{R}_{\xi}^{*}\right)^{2-\eta}=\mathcal{C}_{\chi} /\left(\mathcal{C}_{\xi} / \beta_{\mathrm{c}}^{1 / 2}\right)^{2-\eta}$ and $\mathcal{B}=$ $a_{\chi}-(2-\eta) a_{\xi}$. We also note that the ratios $a_{\xi} / a_{\chi}$ are universal and are known to be about $0.7^{5,19}$. This means that $\mathcal{B} \sim-0.40 a_{\chi}$.

\section{3D SIMPLE CUBIC ISING FERROMAGNET}

For the $3 d$ simple cubic Ising case $N=1$, together with the high precision $\mathrm{MC}$ data at temperatures close

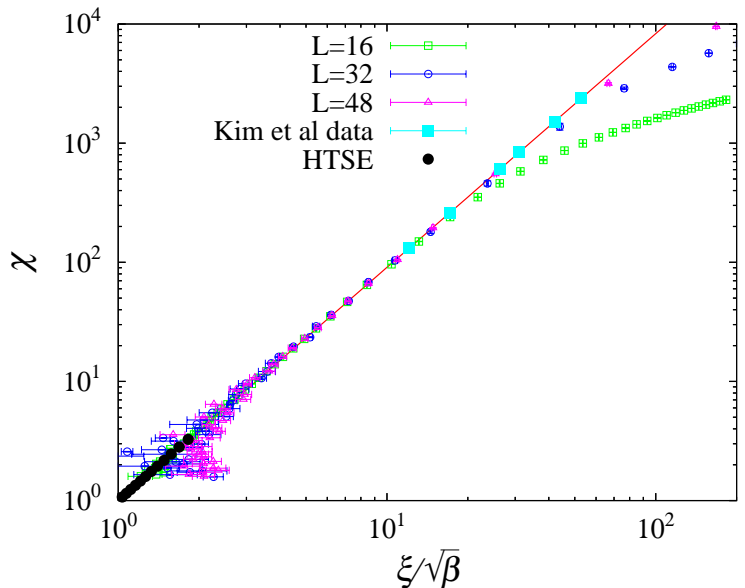

FIG. 1: An extended scaling plot of $\chi$ against $\xi / \sqrt{\beta}$ in the $3 d$ Ising ferromagnet. The filled squares represent the high precision MC data by Kim et $\mathrm{al}^{16}$ and the filled circles numerical estimates from the HTSE of Butera and Comi ${ }^{12}$. Monte Carlo data with $L=16,32$ and 48 by ourselves are also shown. The straight line has a slope of $2-\eta$ with $\eta=0.037(1)$. In this and the following figures our MC data are finite size limited for $T$ close to $T_{c}$, particularly in the case of $L=16$.

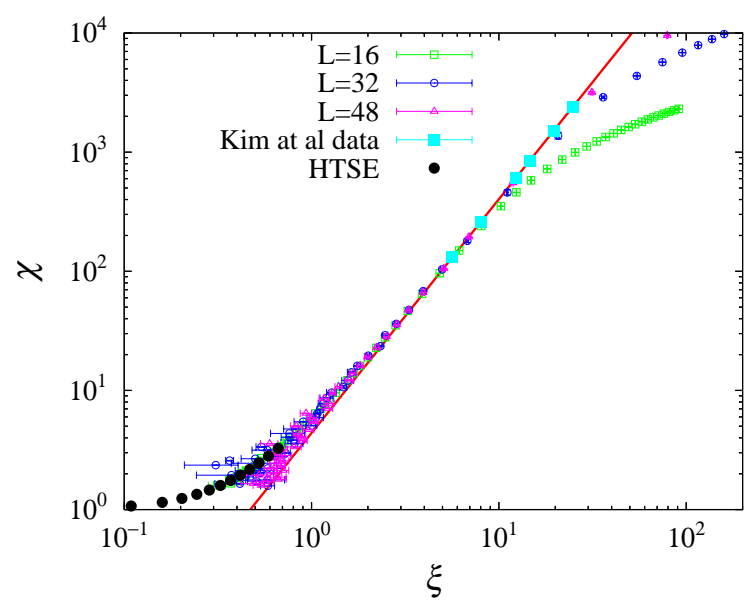

FIG. 2: A conventional scaling plot of $\chi$ against $\xi$ in the $3 d$ Ising ferromagnet. The data are the same as in Fig. 1]

to $T_{\mathrm{c}}$ by Kim et al16 and the HTSE estimates at relatively high temperatures by Butera and Comi $\stackrel{12}{ }$, our own MC data are also used in order to interpolate them and to see overall temperature dependences of $\chi, \xi$ and $C$. In our simulation we used the exchange MC method in combination with 64 bit multi-spin coding technique for making equilibration fast. The 64 different temperatures simulated are distributed in the range of $4.0 \leq T / J \leq 15.0$. The amount of total MC steps for $L=48$ is $2.4 \times 10^{5}$ and the last $8 \times 10^{4} \mathrm{MC}$ steps are used for taking thermal averages.

Figure 1 shows the parameter free log-log plot in the extended scaling form of the reduced susceptibility $\chi$ against $\xi / \sqrt{\beta}$ data. Without allowing for corrections, 
the slope of the line fitted to the data points (ignoring our MC data when they are polluted by finite-size effects) gives a first estimate $\eta \sim 0.037$. Figure 2 is the equivalent standard ( $T$ or $\beta$ scaling) $\log -\log$ plot of $\chi$ against $\xi$ with the slope fixed to the one obtained from Fig. 1. It can be seen that in the standard scaling form the linear relationship breaks down rather quickly while in the extended scaling form with the same input data, the linearity persists to a good approximation up to an infinite temperature and down to temperatures near $T_{c}$ until limited by finite-size effects.

We examine the leading correction of the extended scaling formula given by Eq. (35). To higher precision, Fig. 3 shows a plot of $\chi /(\xi / \sqrt{\beta})^{2-\eta}$ against $\tau^{\theta}$, assuming $\beta_{\mathrm{c}}=0.2216544, \eta=0.0368$ and $\theta=$ $0.504^{7.20}$. The line is obtained by fitting the data points at $\tau^{\theta} \leq 0.4$ to Eq. (35). The intercept at $\tau=0$, $\mathcal{R}^{*}=0.971(4)$, is in good agreement with the value $\mathcal{C}_{\chi} /\left(\mathcal{C}_{\xi} /\left(\beta_{\mathrm{c}}\right)^{1 / 2}\right)^{2-\eta}=0.9767(20)$ assuming the critical amplitudes from HTSE 19 . From the initial slope, $\mathcal{B}=$ $a_{\chi}-(2-\eta) a_{\xi}=0.086(11)$, which we will comment on below.

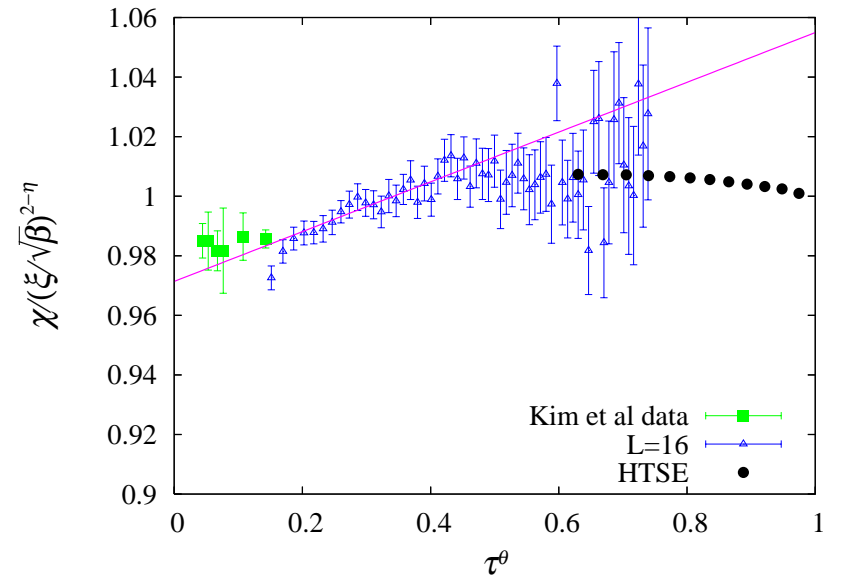

FIG. 3: A plot of $\chi /(\xi / \sqrt{\beta})^{2-\eta}$ against $\tau^{\theta}$ in the $3 d$ Ising ferromagnet. The values of the critical parameters are assumed as described in the text. The straight line is the fit to Eq. (35) with $\mathcal{R}^{*}=0.971$ and $\mathcal{B}=0.086$.

Figures 4 and $\left[5\right.$ show the ratios $R_{\chi}(\tau)$ and $R_{\xi}(\tau)$ of Eqs. (16) and (20), respectively. The numerical data are taken from Kim et al ${ }^{16}$, and the higher temperature values are calculated using the tabulated series of Butera and Comi ${ }^{12}$. The HTSE terms were simply summed, and the points quoted correspond to the temperature range where the contributions from further terms can be considered negligible on the scale of the plots. By using appropriate extrapolation techniques, like differential approximations, the range over which the published HTSE data ${ }^{12}$ could be used to evaluate the temperature dependence of the observables to high precision could be considerably extended. The assumed critical parameters are $\gamma=1.2372, \nu=0.6302$ and $\theta=0.504 \underline{20}$. From the initial intercepts and slopes of the fitted line at small $\tau$, we obtain $\mathcal{R}_{\chi}^{*}=1.132(6), \mathcal{R}_{\xi}^{*}=1.074(3)$, $a_{\chi}=-0.138(23)$ and $a_{\xi}=-0.109(20)$. The $\mathcal{R}_{F}^{*}$ values are in excellent agreement with the HTSE estimates $\underline{19}$, $\mathcal{R}_{\chi}^{*}=\mathcal{C}_{\chi}=1.111(1)$ and $\mathcal{R}_{\xi}^{*}=\mathcal{C}_{\xi} / \beta_{\mathrm{c}}^{1 / 2}=1.0677(7)$. The $a_{F}$ values are in qualitative agreement with the HTSE estimates $a_{\chi}=-0.10(3)$ and $a_{\xi}=-0.12(3) \stackrel{19}{\underline{19}}$.

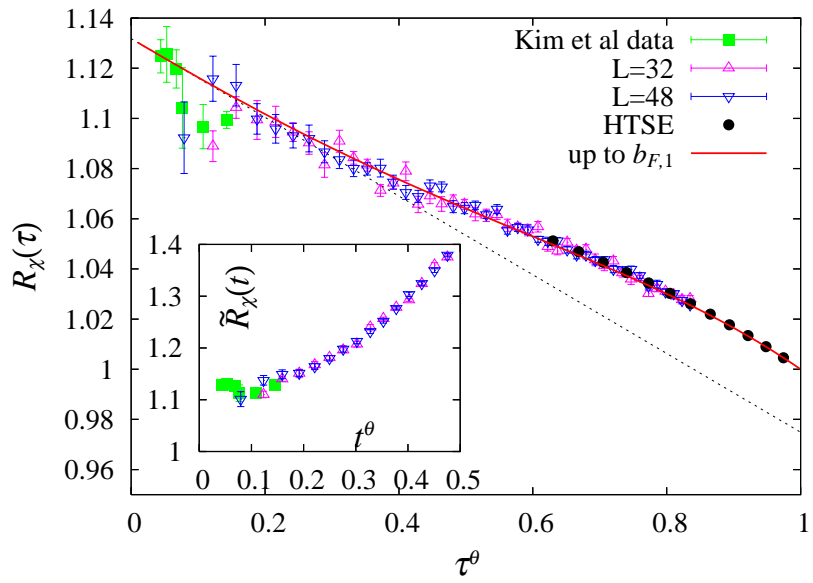

FIG. 4: A plot of the ratio $R_{\chi}(\tau)$ against $\tau^{\theta}$ in the $3 d$ Ising ferromagnet. The straight line represents a fitting to $R_{\chi}(\tau)=$ $\mathcal{R}_{\chi}^{*}\left(1+a_{\chi} \tau^{\theta}\right)$ with $\mathcal{R}_{\chi}^{*}=1.132(6)$ and $a_{\chi}=-0.138(23)$, while the curve does $R_{\chi}(\tau)$ calculated from $\chi^{\text {opt }}(\beta)$ of Eq. (9). In the inset, the $T$ scaling ratio $\tilde{R}_{\chi}(t)$ against $t^{\theta}$ is shown.

An overall conclusion on the extended scaling analysis of the $3 d$ simple cubic Ising data, which will be confirmed by the analyses of the two other systems as well, is that this form of scaling is entirely consistent with the high precision values of critical parameters from extensive HTSE and field theoretical (FT) work. It is remarkable that over the entire temperature range from $T_{\mathrm{c}}$ to

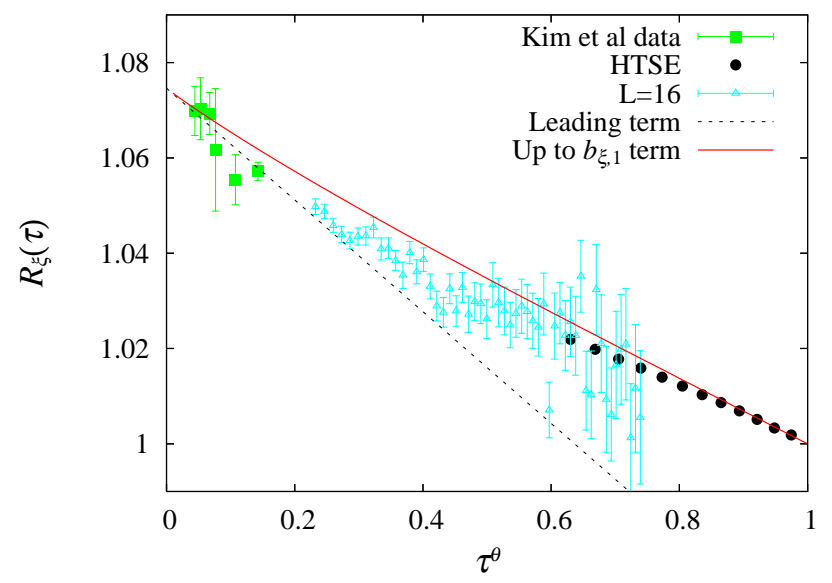

FIG. 5: A plot of the ratio $R_{\xi}(\tau)$ against $\tau^{\theta}$ in the $3 d$ Ising ferromagnet. The broken straight line represents a fitting the data by $\mathrm{Kim}$ et al. to $R_{\xi}(\tau)=\mathcal{R}_{\xi}^{*}\left(1+a_{\xi} \tau^{\theta}\right)$ with $\mathcal{R}_{\xi}^{*}=$ 1.074(3) and $a_{\xi}=-0.109(20)$, while the curve does $R_{\xi}(\tau)$ calculated from $\xi^{\mathrm{opt}}(\beta)$ of Eq. (9). 
infinity, the maximum deviations from the leading critical expressions of Eqs. (15) and (18) are of the order of a few percent. Let us go into further discussions about the $R_{\chi}(\tau)$ behavior. In the inset of Fig. 4 we show the corresponding $T$ scaling ratio $\tilde{R}_{\chi}(t) \equiv \chi(T) / t^{-\gamma}$ plotted against $t^{\theta}$. The latter is calculated using the same values of the critical parameters $T_{\mathrm{c}}, \theta, \gamma$ and $\mathcal{C}_{\chi}$ as those for $R_{\chi}(\tau)$, and so by construction in the low $t, \tau$ limit the intercepts and slopes of both ratios must coincide. It can be seen that in fact the $T$ scaling curve only approaches the $\beta$ scaling curve closely in the range of $t, \tau$ extremely close to zero. This result for $\chi$ with $\phi=0$ strongly suggests the superiority of the $\beta$ scaling, and hence our extended scaling, over the $T$ scaling.

The full curve in the main frame of Fig. 目 is the optimized expression, $R_{\chi}^{\text {opt }}(\tau)$, which is evaluated through $\chi^{\mathrm{opt}}(\tau)$, with one confluent correction term discussed above and the two non-critical terms. The first term of the latter is a constant, $b_{\chi, 0}=1-\mathcal{C}_{\chi}$, which yields simply $R_{\chi}^{\text {opt }}=1$ at $\tau=1$, or at an infinite temperature. Its second term $b_{\chi, 1}$, which is also calculated via the parameters already fixed, specifies the slope of $R_{\chi}^{\text {opt }}(\tau)$ at $\tau=1$. By taking into account only these three correction terms to the leading critical term, $\chi_{\mathrm{c}}^{*}(\beta)$ of Eq. (15), we obtain $R_{\chi}^{\text {opt }}(\tau)$ which reproduces surprisingly well the real data in the whole temperature range $0 \leq \tau \leq 1$. Notice that $\tau^{\theta}=0.6$ corresponds to $T \simeq 1.57 T_{\mathrm{c}}$. This result also indicates the superiority of our extended scaling with the $\beta$ scaling: $\chi_{\mathrm{c}}^{*}(\beta)$ not only represents the critical behavior of $\chi(\beta)$ close to $T_{\mathrm{c}}$ but also $\chi(\beta)$ in the whole temperature range up to infinity. In this context we note again that $\phi=1$ for the "true" susceptibility and that the reduced susceptibility $\chi(\beta)$ is derived through our extended scaling scheme. We also note that the similarity between the $R_{\chi}(\tau)$ plot in Fig. 4 and the $R_{\xi}(\tau)$ plot in Fig. 5 over the entire range of temperature is striking.

Lastly, Fig. [6] shows $C(\beta) / \beta^{2}$ as a function of $1-\beta^{2} / \beta_{\mathrm{c}}^{2}$. The data points are calculated from the HTSE of Arisue and Fujiwara which extends to powers up to $2 n=46 \underline{\underline{13}}$, MC energy data at $L=128$ and $96^{\underline{11}}$, and our numerical simulations for different sizes up to $L=48$. We examine the extended scaling with non-critical contributions to $C(\beta)$ given by Eq. (26). By using the hyper-universal relation with the value of $\mathcal{C}_{\text {hyper }}$ equal to $0.2664(1)^{3}$ for the $3 d$ Ising model and our $\xi$ analysis, we obtain $\mathcal{R}_{C}^{*} \simeq 29.4$. Then the non-critical parameters $\mathcal{K}_{2}$ and $\mathcal{K}_{4}$ are determined by $c_{2}$ and $c_{4}$ of HTSE and with putting $a_{C} \simeq 0$. The solid curve represents the no-free parameter plot of Eq. (26) with the $\alpha, \mathcal{R}_{C}^{*}, c_{2}, c_{4}$ values cited or estimated above. The agreement over the whole temperature range is very satisfactory; the non-critical correction is so strong that the bare leading power law is a poor approximation until very much closer to $T_{\mathrm{c}}$ than the range covered by the figure. We consider this result as an indication that the extended scaling scheme combined with the optimized introduction of correction terms is an effective method for analyzing critically-divergent quantities in general.

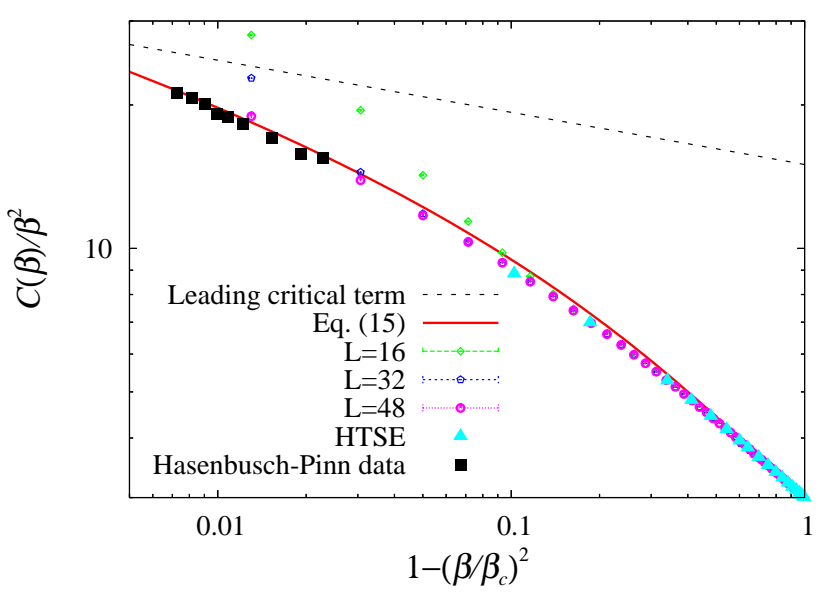

FIG. 6: A plot of $C(\beta) / \beta^{2}$ against $1-\beta^{2} / \beta_{\mathrm{c}}^{2}$. The filled triangles represent the numerical estimates by HTSE of Ref. [13] and the filled squares the MC data of Ref. [11]. The solid line represents the expression of Eq. (26) with $\mathcal{R}_{C}^{*}=29.4$ and $a_{C}=0.1$. The straight broken line is the bare leading critical power law as $\left(1-\left(\beta / \beta_{\mathrm{c}}\right)^{2}\right)^{-\alpha}$.

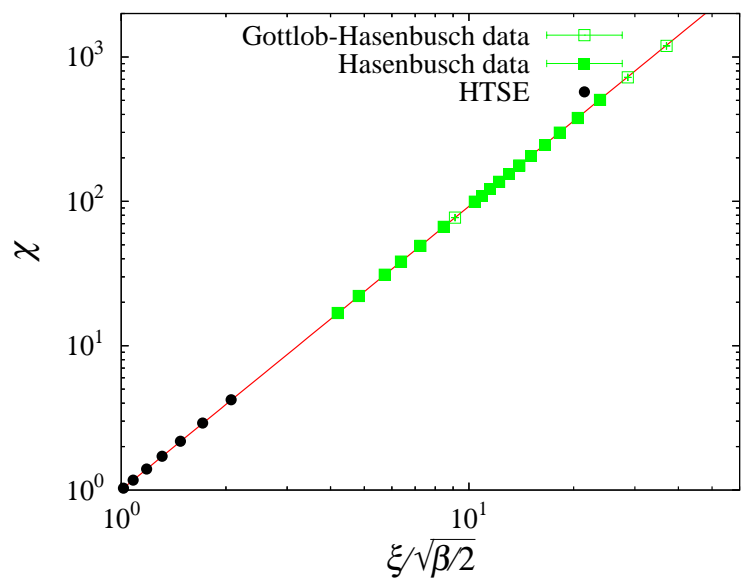

FIG. 7: An extended scaling plot of $\chi(\beta)$ against $\xi / \sqrt{\beta / 2}$ in the $3 d \mathrm{XY}$ ferromagnet. The squares represent the high precision MC data by Gottlob and Hasenbusch ${ }^{17}$ and Hasenbusch $^{21}$, and the filled circles the numerical estimates by HTSE of Butera and Comi ${ }^{12}$. The fitted straight line has a slope of $2-\eta$ with $\eta=0.036$.

\section{V. $3 d$ XY SIMPLE CUBIC FERROMAGNET}

The same analysis has been carried out for the $3 d \mathrm{XY}$ model $(N=2)$. High precision numerical data were published by Gottlob and Hasenbusch $\frac{17}{}$, and are supplemented here by unpublished data kindly provided by M. Hasebusch ${ }^{21}$. The higher temperature data are calculated using the tabulated series of Butera and Comi $\underline{19}$. The critical point is $\beta_{\mathrm{c}}=0.4541652(5)$ and the exponents $\eta, \theta, \gamma$ and $\nu$ are close to $0.0381,0.53,1.3178$ and 0.6717 , respectively $7,8,19$. Figure 7 shows the $\chi-\xi /(\beta / 2)^{1 / 2} \log$ $\log$ plot. The leading scaling scheme works well up to 


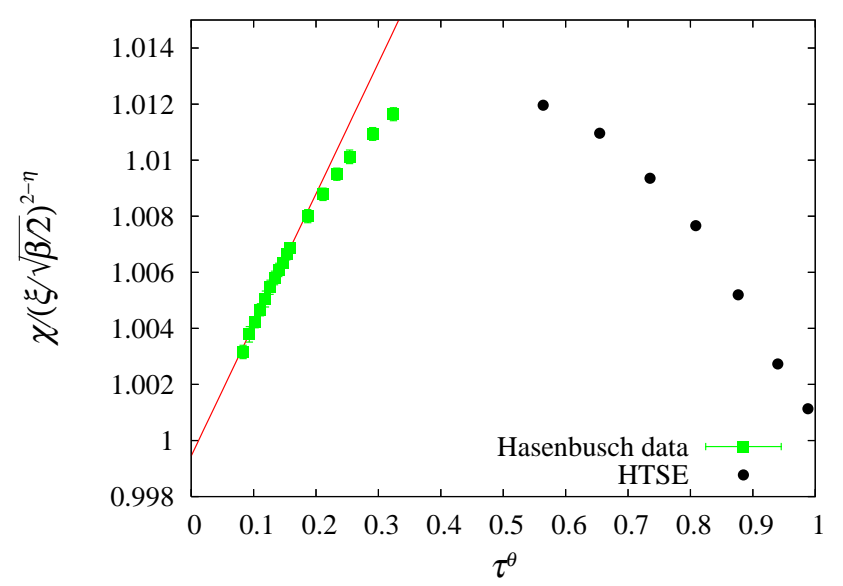

FIG. 8: A plot of $\left.\chi /(\xi / \sqrt{\beta / 2})^{2-\eta}\right)$ against $\tau^{\theta}$ in the $3 d \mathrm{XY}$ ferromagnet. The critical parameters are assumed as $\beta_{\mathrm{c}}=$ $0.4541652, \eta=0.0381$ and $\theta=0.53$. The solid line shows a fitting line to Eq. with $\mathcal{R}^{*}=0.9995(2)$ and $\mathcal{B}=0.047(1)$.

very high temperatures, as in the Ising case. The slope in Fig. 7 gives us the value of $\eta$ which is in agreement with the previously reported values 22 . Figure 8 shows the plot of $\chi(\tau) /[\xi(\tau) / \sqrt{\beta / 2}]^{2-\eta}$ against $\tau^{\theta}$ assuming the central values for the exponents $\eta$ and $\theta$ as mentioned above. Figures 9 and 10 show $R_{\chi}(\tau)$ and $R_{\xi}(\tau)$ respectively against $\tau^{\theta}$. From the $\tau=0$ intercept and the initial slope one can estimate $\mathcal{R}_{\chi}^{*}=1.0471(4), \mathcal{R}_{\xi}^{*}=1.0238(3)$, $a_{\chi}=-0.093(3)$ and $a_{\xi}=-0.073(2)$. These are all reasonably close to the quite independent HTSE values 19 $\mathcal{R}_{\chi}^{*}=1.014(1), \mathcal{R}_{\xi}^{*}=1.0102(6), a_{\chi}=-0.04(2)$ and $a_{\xi}=-0.07(3)$, but are probably more reliable as they are consistent with the independent FT estimate of the universal ratio $a_{\xi} / a_{\chi} \sim 0.65$, see comments in Ref. [19]. Also, the values of $R^{*}$ and $\mathcal{B}$ in Eq. (35) calculated from thus obtained set of the parameters reproduce well the data as shown in Fig. 8, This agreement again validates the extended scaling protocol and demonstrates that a combination of information from FT, HTSE, and simulations analyzed using this protocol can lead to consistent high precision critical parameter measurements.

For comparison, we plot the standard $T$ scaling ratio $\tilde{R}_{\chi}(t)$ introduced in Sec. IV also in Fig. 9, Its coincidence with $R_{\chi}(\tau)$ will only hold for $t \ll 0.01$. As is the case for the Ising system, the slope of $\tilde{R}_{\chi}(t)$ is opposite to that of $R_{\chi}(\tau)$ and the magnitude of $\tilde{R}_{\chi}(t)-\tilde{R}_{\chi}(0)$ is much larger than the corresponding magnitude of the extended ratio already at $t^{\theta}, \tau^{\theta} \sim 0.2$, or $t, \tau \sim 0.04$. In Fig. 10, we also show the $T$ scaling $\tilde{R}_{\xi}(t)=\left(\xi(T) / \sqrt{\beta_{\mathrm{c}} / 2}\right) / t^{-\nu}$ and the $\bar{R}_{\xi}(\tau)=\left(\xi(T) / \sqrt{\beta_{\mathrm{c}} / 2}\right) / \tau^{-\nu}$ by $\beta$-scaling. The true leading term plus confluent correction holds with the extended scaling form, $R_{\xi}(\tau)$ of Eq. (20) with $N=2$ up to $t \sim 0.1$ while with the other forms of scaling the correct limit will hold only for $t \ll 0.01$. In particular, the comparison of $\beta$ scaling $\bar{R}_{\xi}(\tau)$ with extended scaling $R_{\xi}(\tau)$ demonstrates the importance of the $\beta^{1 / 2}$ prefac-

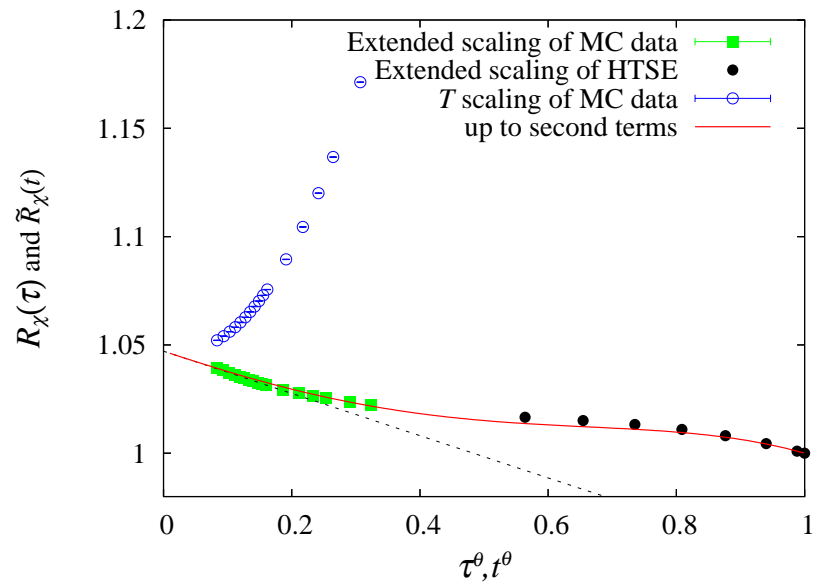

FIG. 9: A plot of the ratio $R_{\chi}(\tau)$ against $\tau^{\theta}$ in the $3 d \mathrm{XY}$ ferromagnet. The value of $\gamma$ is assumed to be $\gamma=1.3178^{8}$. The filled marks are obtained by the extended scaling of $\operatorname{HTSE}^{12}$ and the MC data 21 . The broken line represents a fitting to $R_{\chi}(\tau)=\mathcal{R}_{\chi}^{*}\left(1+a_{\chi} \tau^{\theta}\right)$ with $\mathcal{R}_{\chi}^{*}=1.0471(3)$ and $a_{\chi}=-0.093(3)$, while the curve does $R_{\chi}(\tau)$ calculated from $\chi^{\mathrm{opt}}(\beta)$ of Eq. (9). The standard $T$-scaling ratio $\tilde{R}_{\chi}(t)$ as a function of $t^{\theta}$ is also plotted by the open circles.

tor in Eq. (20) of the extended scaling scheme. These results imply that even close to $T_{\mathrm{c}}$ the extended scaling is a considerable improvement over the standard scaling analysis for estimating critical parameters including the correction terms.

The curve in Fig. 9 represents our optimized estimates $R_{\chi}^{\text {opt }}(\tau)$ up to the second order of non-critical corrections. It reproduces about 5 percents change in $R_{\chi}(\tau)$, from about 1.05 at $\tau=0$ to 1 at $\tau=1$, to a very good approximation. The corresponding relative change in $R_{\xi}(\tau)$ is only less than 2 percents as seen in Fig. 10. To reproduce this change by $R_{\xi}^{\mathrm{opt}}(\tau)$ to an approximation as good as $R_{\chi}^{\text {opt }}(\tau)$ in Fig. 9] however, more than third order noncritical correction terms are required.

\section{VI. $3 d$ HEISENBERG SIMPLE CUBIC FERROMAGNET}

The same analysis has been carried out for the $3 d$ Heisenberg model $(N=3)$. High precision numerical data were published by Holm and Janke $\underline{18}$, and are supplemented here by higher temperature data calculated using the tabulated series of Butera and Comi $\underline{19}$. The critical point is $\beta_{\mathrm{c}}=0.69305(4)$ and the exponents $\eta$ and $\theta$ are close to 0.036 and $0.55^{7.19}$. A recent exponent set ${ }^{22}$ gives $\gamma=1.3960(9), \nu=0.7112(5)$ and $\eta=0.0375(5)$.

Figure 11 shows the $\chi(T)-\xi(T) / \sqrt{\beta / 3}$ log-log plot, which gives an estimate of $\eta$ consistent with that of Ref. 22]. Figure 12 shows the plot of $\chi(T) /[\xi(T) / \sqrt{\beta / 3}]^{2-\eta}$ against $\tau^{\theta}$ assuming the exponent values as $\eta=0.0375$ and $\theta=0.55$. From this plot it 


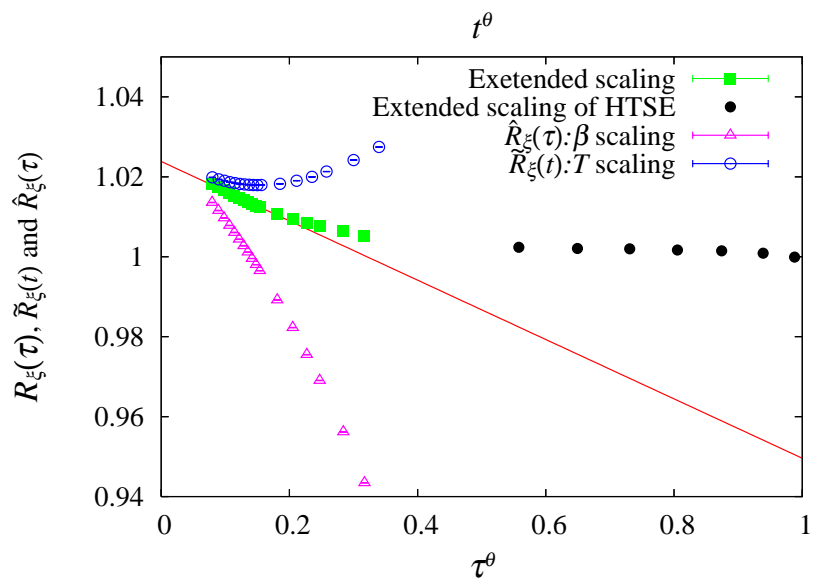

FIG. 10: A plot of the ratio $R_{\xi}(\tau)$ against $\tau^{\theta}$ in the $3 d \mathrm{XY}$ ferromagnet. The value of $\nu$ is assumed to be $\nu=0.6717$. The line represents a fitting to $R_{\xi}(\tau)=\mathcal{R}_{\xi}^{*}\left(1+a_{\xi} \tau^{\theta}\right)$ with $\mathcal{R}_{\xi}^{*}=1.0238(3)$ and $a_{\xi}=-0.073(2)$. The ratios from $T$ scaling $\tilde{R}_{\xi}(t)$ and $\beta$-scaling $\hat{R}_{\xi}(\tau)$ are shown by open circles and open triangles, respectively.

appears that the initial slope is very small, corresponding to almost zero values for $a_{\chi}$ and $a_{\xi}$. Figures 13 and 14 show respectively $R_{\chi}(\tau)$ and $R_{\xi}(\tau)$ against $\tau^{\theta}$, assuming the values of $\gamma$ and $\nu$ in Ref. 22]. The MC and HTSE points may not appear to connect smoothly in these figures, because the manner in which the plots are presented enhances small deviations from the leading term form. However, the change in the values of both $R_{\chi}(\tau)$ in Fig. 13 and $R_{\xi}(\tau)$ in Fig. 14 are limited to within a few percent of their absolute magnitude in a whole range of $\tau$ as is the case for the other two ferromagnets studied. From the straight line fit of the MC data at small $\tau^{\theta}$, one can estimate $\mathcal{R}_{\chi}^{*}=0.952(2)$, $\mathcal{R}_{\xi}^{*}=0.967(2), a_{\chi}=-0.04(1)$ and $a_{\xi}=-0.03(2)$. In this case the parameters are slightly less consistent with the HTSE estimates $\frac{19}{}, \mathcal{R}_{\chi}^{*}=0.9030(8), \mathcal{R}_{\xi}^{*}=0.9447(5)$, $a_{\chi}=0.06(3)$ and $a_{\xi}=0.003(6)$, but it should be noted that the estimates for these [non-universal] parameters depend very sensitively on the precise values taken for the critical exponents. We certainly need more precise data near $T_{\mathrm{c}}$ to fix the values of these critical parameters for the Heisenberg ferromagnet.

\section{CONCLUSION}

We have outlined a systematic rule for the scaling and normalization of thermodynamic observables having critical behavior at continuous phase transitions. This "extended scaling" rule corresponds for ferromagnets to scaling of the leading term of the reduced susceptibility above $T_{\mathrm{c}}$ as $\chi_{\mathrm{c}}(T)=\mathcal{R}_{\chi}^{*} \tau^{-\gamma}$ in agreement with standard practice, for the leading term of the second moment correlation length as $\xi_{\mathrm{c}}(T)=\mathcal{R}_{\xi}^{*} \beta^{1 / 2} \tau^{-\nu}$ with $\mathcal{R}_{\xi}^{*}=$

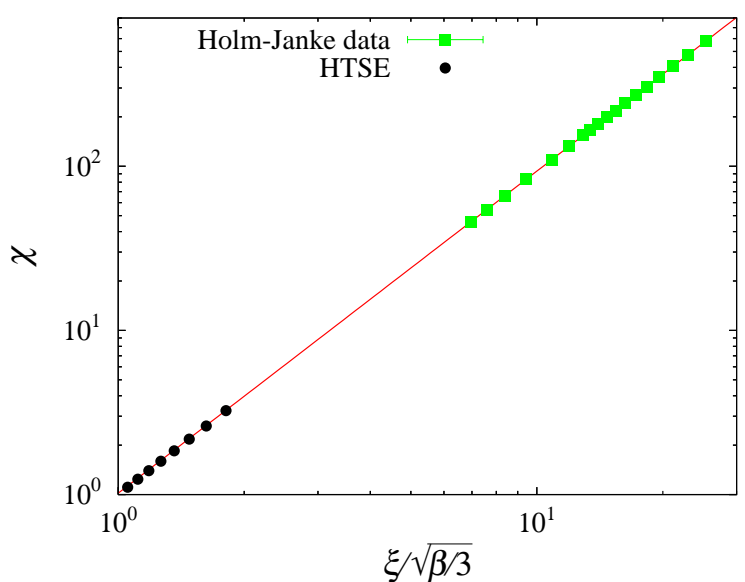

FIG. 11: An extended scaling plot of $\chi(\beta)$ against $\xi / \sqrt{\beta / 3}$ in the $3 d$ Heisenberg ferromagnet. The filled squares represent the high precision MC data by Holm and Janke ${ }^{18}$ and the filled circles the numerical estimates from the HTSE of Butera and Comi ${ }^{12}$. The straight line has a slope of $2-\eta$ and the best fit gives $\eta=0.0379(4)$.

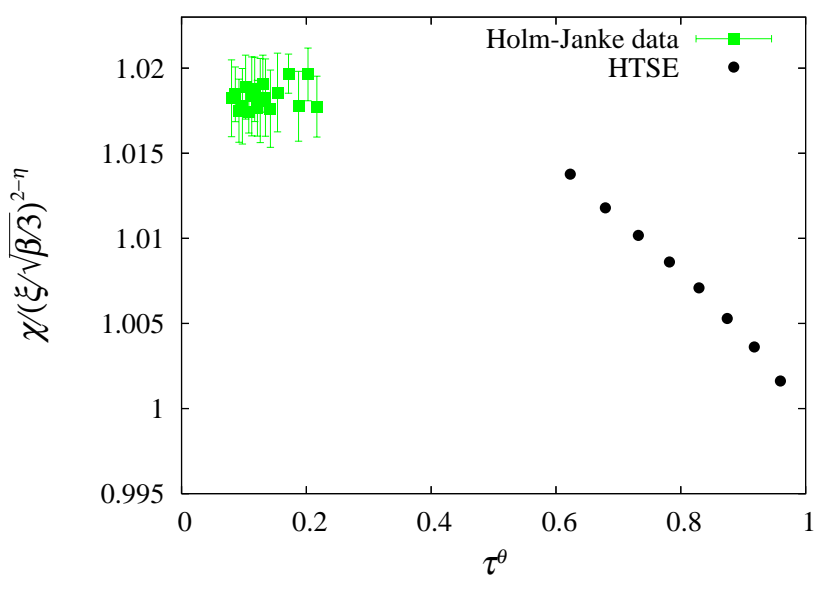

FIG. 12: A plot of $\left.\chi /(\xi / \sqrt{\beta / 3})^{2-\eta}\right)$ against $\tau^{\theta}$ in the $3 d$ Heisenberg ferromagnet. The critical parameters are assumed as $\beta_{\mathrm{c}}=0.69305, \eta=0.0375$ and $\theta=0.55$.

$\mathcal{C}_{\xi} /\left(z \beta_{c} / 2 d N\right)^{1 / 2}$ and for the leading term of the specific heat in bipartite lattices $C(T)=\beta^{2} \mathcal{R}_{C}^{*}\left(1-\left(\frac{\beta}{\beta_{c}}\right)^{2}\right)^{-\alpha}$ with $\mathcal{R}_{C}^{*}=\mathcal{C}_{C} 2^{\alpha} / \beta_{\mathrm{c}}^{2}$ plus strong non-critical correction terms which we explicitly evaluate by linking to the HTSE.

Analyses are made of high precision numerical data on three canonical ferromagnets using these expressions allowing for confluent scaling correction terms, plus noncritical corrections for the specific heat. Near $T_{\mathrm{c}}$ the results are entirely consistent with the critical parameter sets (including the confluent corrections) which have been obtained independently using sophisticated FT, HTSE and simulation techniques $7,19,20,22$. 


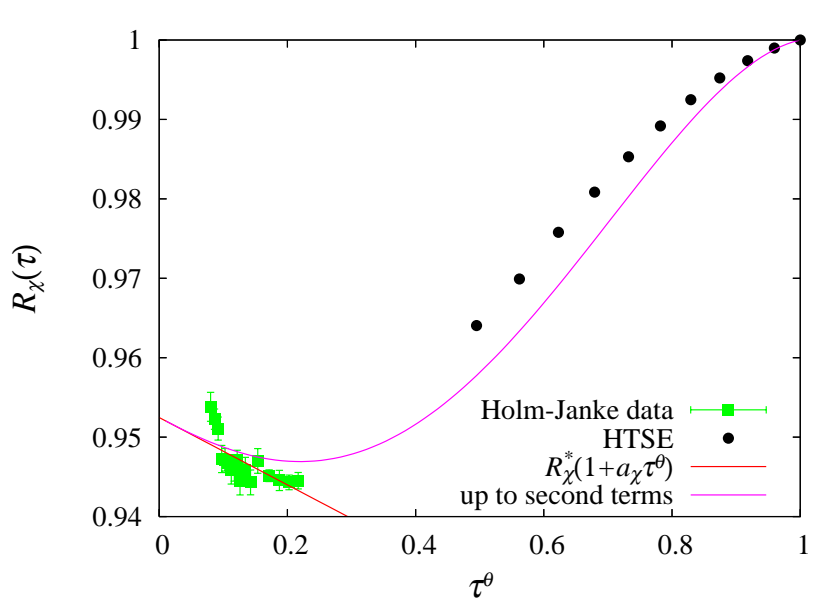

FIG. 13: A plot of the ratio $R_{\chi}(\tau)$ against $\tau^{\theta}$ in the $3 d$ Heisenberg ferromagnet. The straight line represents a fitting to $R_{\chi}(\tau)=\mathcal{R}_{\chi}^{*}\left(1+a_{\chi} \tau^{\theta}\right)$ with $\mathcal{R}_{\chi}^{*}=0.952$ and $a_{\chi}=-0.04$, while the curve does the optimized form using up to the second non-critical correction terms.

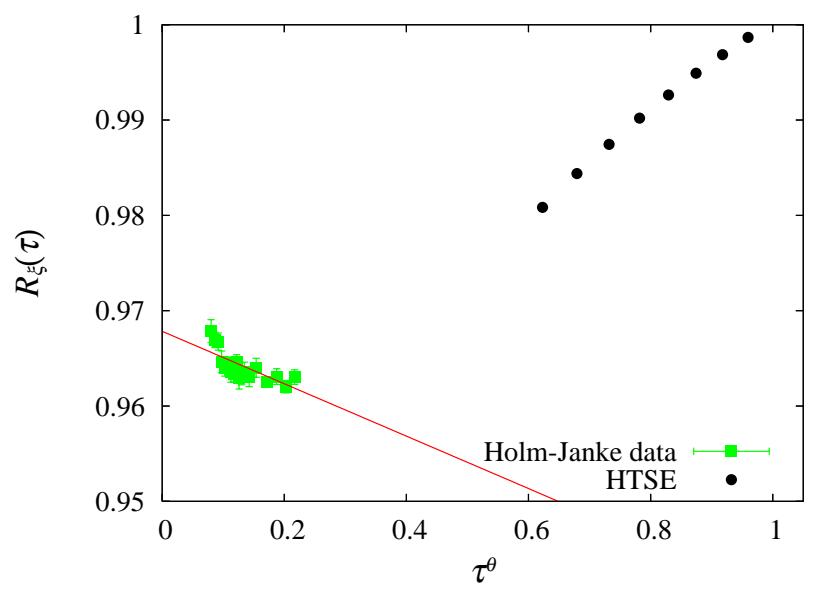

FIG. 14: A plot of the ratio $R_{\xi}(\tau)$ against $\tau^{\theta}$ in the $3 d$ Heisenberg ferromagnet. The line represents a fitting to $R_{\xi}(\tau)=\mathcal{R}_{\xi}^{*}\left(1+a_{\xi} \tau^{\theta}\right)$ with $\mathcal{R}_{\xi}^{*}=0.967$ and $a_{\xi}=-0.03$.

The most important result found in the present work is that, for $\chi(T)$ and $\xi(T)$ the leading critical expressions with the extended scaling normalizations $F_{c}^{*}(\beta)$ of Eq. (8) agree to a very good approximation with the true $F(\beta)$ up to infinite temperature. To demonstrate this fact more in details we have introduced the ratio $R_{F}(\tau)$ defined by Eq. (11). For $\chi$ of the Ising ferromagnet, for example, it is equal to the critical amplitude $\mathcal{C}_{\chi}$ at $T_{\mathrm{c}}$ $(\tau=0)$ and to unity at infinite temperature $(\tau=1)$ by definition. $R_{\chi}(\tau)$ evaluated from the true data are represented by the data points in Fig. 4, while $R_{\chi}(\tau)$ evaluated through the leading expression $\chi_{c}^{*}(\beta)$ is independent of $\tau$ and equal to $\mathcal{C}_{\chi}$. The difference between the two is, however, at most 13 percent in this case. The corresponding differences for $R_{\chi}(\tau)$ 's of the two other ferromagnets as well as for $R_{\xi}(\tau)$ 's of the all three ferromagnets are less than several percent. This is our first result mentioned just above.

We have next demonstrated that our extended scaling scheme, in terms of the $\beta$ scaling and with the temperature dependent prefactor $\beta^{\phi_{F}}$, is of crucial importance in precisely extracting the small amplitude $a_{F}$ of the leading confluent correction term. The result is represented by the solid line in Fig. 4as well as those in Figs. 5, 9, and 10. In addition, we have also checked that the optimized expression $F^{\mathrm{opt}}(\beta)$ of Eq. (9), consisting of $F_{\mathrm{c}}^{*}(\beta)$ and one confluent and two non-critical correction, yield $R_{F}^{\text {opt }}(\tau)$ which reproduces the true $R_{F}(\tau)$ surprisingly well as shown the curves in Figs. 4 and 9, though more than third non-critical correction terms would be required for equally good agreement in other observables.

The large non-critical terms in the specific heat $C(T)$ are also incorporated explicitly within our extended scaling scheme with no further adjustable input parameters. For the Ising ferromagnet on the simple cubic lattice $C(T)$ is calculated to a good approximation over the entire temperature range (see Eq. (26)). Although the noncritical correction terms are large for $C(T)$, the principle of the analysis is the same as the one applied above to $\chi(T)$ and $\xi(T)$, for which the corrections to scaling are quite small. Namely, each critically-divergent observable $F(\beta)$ is represented by $F^{\mathrm{opt}}(\beta)$ of Eq. (9) over the whole range of $\beta$ to a good approximation. The input consists of $F_{c}^{*}(\beta)$, a confluent correction term and a very limited numbers of non-critical correction terms derived from HTSE.

Together these results can be taken as validating the "extended scaling" approach. The approach could be systematically implemented in numerical work so as to improve yet further the accuracy of critical parameter sets derived for standard systems, possibly incorporating where necessary further higher order correction terms.

Perhaps a more fruitful application would concern the analyses of numerical data in more complex systems, where the present accuracy of the critical parameter sets is much poorer. For instance, it has been pointed out that for the analysis of data on spin glasses with symmetric interaction distributions $\beta$ should be replaced by $\beta^{2}$ in all expressions $\frac{6,23}{}$ as all terms in the HTSE in these spin glasses are strictly even in $\beta$. The extended scaling protocol allowing for this and with appropriate $\phi_{F}(\beta)$ normalization factors has indeed been shown to significantly improve the consistency of critical exponent values derived from numerical simulations on Ising spin glasses ${ }^{6.24}$.

\section{Acknowledgments}

We would like to thank P. Butera for all his careful and patient advice, H. Arisue for providing extensive tabulated series data, M. Hasenbusch for allowing us to use his unpublished high-precision numerical data, and W. Janke for helpful discussions. This work was supported by the Grants-In-Aid for Scientific Re- 
search (No. 17540348 and No. 18079004) and NAREGI Nanoscience project, both from MEXT of Japan. The numerical calculations were mainly performed on the SGI
Origin 2800/384 at the Supercomputer Center, ISSP, the University at Tokyo.
1 A. Pelissetto and E. Vicari, Phys. Rept.368 549 (2002).

2 S. Gartenhaus and W. S. McCullough, Phys. Rev. B 38, 11688 (1988).

3 P. Butera and M. Comi, Phys. Rev. B 65, 144431 (2002).

4 F.J. Wegner, Phys. Rev. B 54529 (1972).

5 C. Bagnuls and C. Bervillier, Phys. Rev. B 24, 1226 (1981).

${ }^{6}$ I. A. Campbell, K. Hukushima and H. Takayama, Phys. Rev. Lett. 97, 117202 (2006).

7 R. Guida and J. Zinn-Justin, J. Phys. A 31, 8103 (1998).

8 M. Campostrini, M. Hasenbusch, A. Pelissetto, and E. Vicari, Phys. Rev. B 74, 144506 (2006).

${ }^{9}$ G. Parisi, in Statistical Field Theory, (Perseus Books, 1988).

10 M. E. Fisher and R. J. Burford, Phys. Rev.156, 583 (1967).

11 M. Hasenbusch and K. Pinn, J. Phys. A 31, 6185 (1998).

12 P. Butera and M. Comi, J. Stat. Phys. 109, 311 (2002), hep-lat/0204007.

13 H. Arisue and T. Fujiwara, Phys.Rev. E 67, 066109 (2003).

14 D. Stauffer, M. Ferer and M. Wortis, Phys. Rev. Lett. 29,
345 (1972).

15 M. E. Fisher and M. N. Barber, Phys. Rev. Lett. 28, 1516 (1972).

16 J.K. Kim, A.J.F. de Souza and D.P. Landau, Phys. Rev. E 54, 2291 (1996)

17 A.P. Gottlob and A. Hasenbusch, Physica A 201, 593 (1993).

18 C. Holm and W. Janke, Phys. Rev. B 48, 936 (1993).

19 P. Butera and M. Comi, Phys. Rev. B 58, 11552 (1998).

${ }^{20}$ Y. Deng and H.W.J. Blöte, Phys. Rev. E 68, 036125 (2003).

21 M. Hasenbusch, private communication.

22 M. Campostrini, M. Hasenbusch, A. Pelissetto, P. Rossi, and E. Vicari, Phys. Rev. B 65, 144520 (2002).

23 D. Daboul, I. Chang and A. Aharony, Eur. Phys. J. B 41, 231 (2004).

24 H.G. Katzgraber,M. Körner, and A. P. Young, Phys Rev B 73, 224432 (2006) 